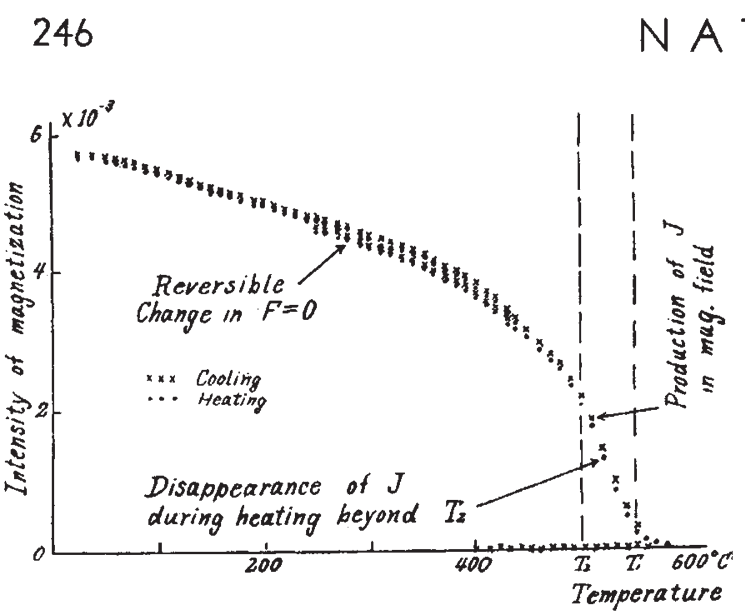

Fig. 2. Development of magnetization by cooling in magnetic field, is reversible change in non-magnetic space. and its disappearance by heating. (Sample: olivine-hypersthene-pyrox-

of the magnetization produced persists as the remanent magnetism. Below $500^{\circ} \mathrm{C}$.. the remanent magnetization $J(t)$ still increases with decrease in temperature even in non-magnetic space, and $J(t)$ changes reversibly with the change in temperature $t$, provided that $t$ does not exceed $5 \cap 0^{\circ} \mathrm{C}$. and no magnetic field is further applied during any process of cooling. The results of a group of similar experiments show that the nucleus of remanent magnetization in rocks produced by cooling from $T_{1}$ to $T_{2}\left(T_{1}>T_{2}\right)$ in a field $F$ always disappears on heating them from $T_{2}$ through $T_{1}$, regardless of the intensity and direction of magnetic field applied during the heating process.

This law holds even when $T_{1}-T_{2}=\Delta T=5^{\circ}$. We may say, then, that some vectors of magnetic domains in the ferro-magnetic minerals are fixed in the direction of the applied magnetic field duing cooling from $T_{1}$ to $T_{2}$ in the magnetic field $F$, while they are released in the reverse process. The reversible change of $J(t)$ with temperature below $T_{2}$ may correspond to the reversible change in the spontaneous magnetization of domains, well known as the Curie law.

If $\varphi(F, T)$ denotes the probability of orientation of domain vectors in the direction of $F$ during cooling through a small temperature-range $\Delta T$ around $T=\left(T_{1}+T_{2}\right) / 2$, and $I(T, t)$ gives the intensity of their spontaneous magnetization at $t$, we get for the magnitude of the corresponding remanent magnetization :

$$
\Delta J=I(T, t) \cdot \varphi(F, T) \Delta T, \quad t<T .
$$

Experiments on a number of rock samples indicate that $O(F, T)$ changes with $T$ in a manner similar to the curve shown in Fig. 1 , and that values of $\Delta J(T, t)$ produced in a rock sample at different values of $T$ are independent of each other. Then, denoting the remanent magnetization at $t$ produced by cooling

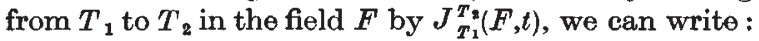

$$
J_{T_{1}}^{T_{2}}(F, t)=\int_{T_{2}}^{T} I\left(T^{\prime}, t\right) \varphi(F, T) d T,\left(t<T_{\mathbf{2}}<T_{1}\right),
$$

where $\varphi(F, T)$ takes place only during the cooling process and vanishes during the heating process. When $F$ is small ( $\leqslant 1$ oersted), it is found that $\varphi(\mathbf{F}, T) \simeq \mathbf{F} . \psi(T)$. Thus,

February 11, 1950 Vol. 165

$$
\mathbf{J}_{T_{1}}^{T_{2}}(F, t) \simeq \mathbf{F} \int_{T_{2}}^{T_{1}} I(T, t) \psi(T) d T .
$$

The other observed facts that $\varphi(F, T)$ is large in rocks of large coercive force, and that the usual A.c. demagnetizing method (up to a magnetic fild 100 times $F$ ) can scarcely remol' $\theta$ the remanent magnetiza. tion produced by cooling in a weak magnetic field, may suggest that $\varphi(F, 1)$ is due to a fairly strong internal stress in the ferromagnetic minerals in rocks.

A similar phenomenon has been recently found in the mignetic properties of $\mathrm{CoO} . \mathrm{Fe}_{2} \mathrm{O}_{3}-\mathrm{Fe}_{3} \mathrm{O}_{4}$, and those of $\mathrm{TiO}_{2} \mathrm{Fe}_{2} \mathrm{O}_{3}-\mathrm{Fe}_{3} \mathrm{O}_{4}$ are now being examined.

My thanks are due to Mr. H. Manley for a useful discussion.

Geophysical Institute,

Takesi Nagata

Tokyo University,

Tokyo.

Sept. 8 .

2 Bruckshaw, E. McG., and Robertsnn, E. I., Mon, Not. Roy. Astro. Soc., Geophys. Supp., 5, 308 (1949). Chapman, S., Nature, 161, $462(19+8)$. 'Koenigsberger, J. G., Terr. Mag., 43, 119 and 299 (1938), and

\section{Partial Ionization in Lithium Hydride}

SINGLE-CRYSTAL rotation photographs of lithium hydride (LiH), using copper $K \propto$ radiation, show clearly that the structure is of a sodium chloride type, but that the lithium and hydrogen atoms are by no means completely ionized. Atomic scattering curves have been drawn from the corrected intensity data for the 'all even' reflexions $(\mathrm{Li}+\mathrm{H})$; and for the 'all odd' reflexions $(\mathrm{Li}-\mathrm{H})$. From these the separate atomic scattering curves for the $\mathrm{Li}^{+} x$ ion and for the $\mathrm{H}^{-x}$ ion (uncorrected for temporature scattering) have been deduced. The thermal scattering, which is of the same order as that in sodium chloride (though differently distributed), has also been studied separately by means of 'diffuse' Laue pliotographs. The results show that the most probable state of ionization is $\mathrm{Li}^{+0}{ }^{25}, \mathrm{H}^{-0}{ }^{25}$. The absolute limits that could be supposed to agree with the observations would be 50 per cent ionic character or, at the opposite extreme, complete covalency.

I am indebted to Prof. Kathleen Lonsdale for help and advice in this research, which will be published more fully later.

University College,

M. S. AhMed

London.

Nov. 11.

\section{Action of Phenolic Substances on the Blood- clotting Effect of Bothrops atrox Venom}

Gerber and Blanchard ${ }^{1}$ reported the accelerating effect of different phenols on blood clotting. Laki et $a l .{ }^{2}$ have also found that catechol added to thrombin shortens the coagulation time of plasma.

During a series of experiments which were carried out for the purpose of comparing the blood-clotting effect of Bothrops atrox venom and thrombin, the action of certain phenolic substances on the coagulation has also been investigated. The differences between the two coagulating principles have been 